\title{
A Current Review on the Pathogenesis and Treatments of Vitiligo
}

\section{IJCRR}

Section: Healthcare

Sci. Journal Impact

Factor: 6.1 (2018)

ICV: 90.90 (2018)

(c) (7) (5)

Copyright@IJCRR

\section{Debosree Ghosh ${ }^{1}$, Suvendu Ghosh ${ }^{2}$}

\begin{abstract}
'Department of Physiology, Government General Degree College, Kharagpur 11, Madpur, Paschim Medinipur 721149, West Bengal, India;
\end{abstract} 2Department of Physiology, Hooghly Mohsin College, Chinsura, Hooghly 712 101, West Bengal, India.

\section{ABSTRACT}

Vitiligo is recognized as an acquired autoimmune depigmentation disorder. Normally, the disease occurs before forty years of age but it has been reported to be effecting people irrespective of age and sex. Body produces antibodies against melanocytes and destroys them. As a result, white patches appear in the body. Vitiligo may be segmental or non-segmental. Objectives of this review article is to understand the underlying mechanism of occurrence of vitiligo, brief an updated information about the available treatments and the possible precautionary measures against vitiligo. All available literature about vitiligo and it's treatment procedures were studied and thus all available information has been reviewed and briefed in this article to represent a compact scenario of the pathogenesis and treatments of vitiligo to make the understanding of the disease clear. Vitiligo has significant social and psychological impacts and physiological adverse effects like hypopigmentation or depigmentation in the patient. The social and psychological impact that the disease brings with it is severe in most cases. Vitiligo is not completely curable but treatments include topical corticosteroids, immune-modulators, and UV therapy which causes repigmentation of white patches, micro pigmentation etc.

Key Words: Autoimmune, Depigmentation, Hypopigmentation, Melanocytes, Vitiligo

\section{INTRODUCTION}

Vitiligo is an autoimmune disorder in which depigmentation of the skin occurs and white patches appear in different parts of the body. In vitiligo, both humoral and cell-mediated immune system work hand in hand and destroy melanocytes. ${ }^{1}$ Several studies have been conducted all around the world to understand the etiology of the disease. It is possible to fight back disease or find out an effective defence against any particular disease only and only if the detailed causative mechanism of the disease is comprehended. So far, considerable progress has been made in understanding the vitiligo and underlying mechanism of the disease. Investigations have revealed various causes and mechanism of occurrence of the disease. The disease has been surely recognized as an autoimmune disorder. It is a chronic pathological condition. Currently, vitiligo has been topographically classified into segmental, zosteriform \& nonsegmental, areata, vulgaris, acrofacialis and mucosal. ${ }^{2}$ Some people experience itching before a new patch appears. ${ }^{3}$ Studies show that white patches of vitiligo are often surrounded by a prominent pigmented border which is termed as the "trichrome vitiligo. ${ }^{2}$ Vitiligo has been found to have morphological variations. The various morphological variations of vitiligo are trichrome, quadri-chrome, penta-chrome, blue and inflammatory vitiligo. ${ }^{2}$ There is no specific treatment to cure the condition. But there are some medicines and ointments that can restrict the spread of the white patches. Around $1 \%$ of people around the world is the victim of vitiligo. ${ }^{3}$ Studies have shown that stress to melanocytes leads to vitiligo. ${ }^{4}$ Mechanical stimulus or chemical exposure or sunburn may induce stress to melanocytes thus altering their normal physiology. This initiates a cascade of the autoimmune response in which body destroys melanocytes. As a result of this, white patches appear. Nowadays oxidative stress is linked with triggering the autoimmune response which is responsible for the occurrence and spread of vitiligo. ${ }^{5} 90 \%$ of vitiligo patients studied were found to have higher activity of superoxide dismutase (SOD) enzyme compared to normal people establishing the involvement of oxidative stress in vitiligo. ${ }^{4}$ Oxidative stress may also simulate the targeting of melanocyte by promoting antigen presentation. ${ }^{5}$ Interleukin (IL)-17-mediated responses and Interferon

\section{Corresponding Author:}

Dr. Debosree Ghosh, Department of Physiology, Government General Degree College Kharagpur II, P.O: Madpur, Dist: Paschim Medinipur, Pin: 721149, West Bengal, India; Mobile: +91 9830320757; Email: ghoshdebosree@gmail.com

ISSN: 2231-2196 (Print)

Received: 21.05 .2020
ISSN: 0975-5241 (Online)

Revised: 13.07 .2020
Accepted: 03.10 .2020 
(IFN)- $\gamma /$ CXCL10 axis are the two major key players in the autoimmune destruction of melanocytes. ${ }^{4}$ IL17 is involved deeply in the pathogenesis of various autoimmune disorders ${ }^{5}$ and vitiligo is one among them..$^{6-8}$ Vitiligo has been found to coexist with other autoimmune disorders such as Sutton or halo nevus, and malignant melanoma. ${ }^{9}$ Details about the available treatments of vitiligo have been discussed in the later part of our review article.

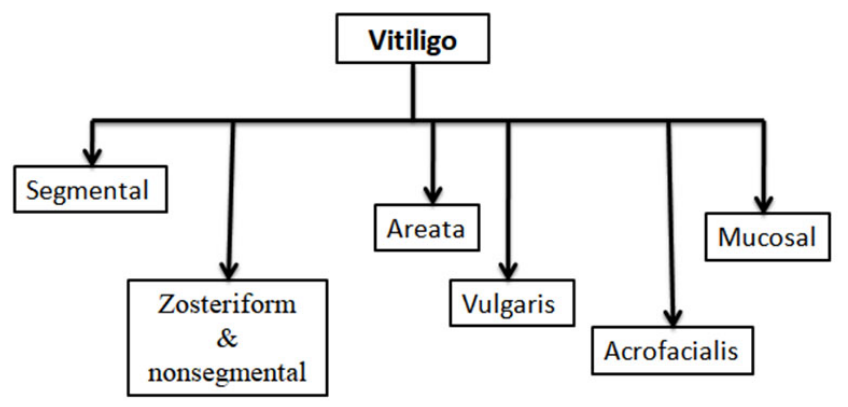

Figure 1: Classification of Vitiligo.

\section{Oxidative stress and vitiligo}

Studies reveal that exposure to certain stimulants such as sunlight, chemicals etc., induce oxidative stress in cells which renders the melanocytes susceptible to destruction by autoimmune responses. Thus vitiligo may be caused due to exposure to certain chemicals and even sunlight or anything that may induce oxidative stress in the melanin-containing cells in our skin. ${ }^{10}$ Genetic polymorphism of the Nrf2 promoter region is associated with vitiligo risk in Chinese populations. ${ }^{11} \mathrm{~A}$ significant association between single nucleotide polymorphism (SNP) rs3565214 within NRF2 and vitiligo has been reported in a study conducted on Chinese population. ${ }^{11} \mathrm{Nrf2}$ is a basic leucine zipper (bZIP) protein which is known to regulate the expression of antioxidant proteins that protect against oxidative damage mediated by oxidative stress induced by injury and inflammation.

The indigenous antioxidant enzyme SOD is linked to vitiligo. This further confirms the link of oxidative stress with the disease. Studies show that people with vitiligo have a significantly higher level of SOD activity in serum and tissues from perilesional areas. ${ }^{12}$ Oxidative stress has been found to cause overexpression of iHSP70 in the skin of mice. Overexpression of iHSP70 has been found to cause melanocyte loss in mice. ${ }^{13}$ Oxidative stress-mediated damaged and dilated endoplasmic reticulum has also been found in melanocytes from perilesional areas in vitiligo patients. ${ }^{14}$ Endoplasmic reticulum (ER) is a site for maturation and bond formations in proteins. Oxidative stress-mediated damage in ER leads to accumulation of misfolded proteins. This stimulates UPR (unfolded protein response) which initially stops overall protein synthesis, produces chaperons etc. UPR if activated long term, leads to apoptosis. Certain phenolic chemicals which cause vitiligo are known to induce oxidative stress and activate UPR. UPR stimulates the production of IL-6 and IL- 8 cytokines which can attract immune components to the $\operatorname{skin}^{15}$ (Fig.2).

\section{Our immune system and vitiligo}

The autoimmune disorder leads to the destruction of the body's melanin-containing cells which causes vitiligo. Certain autoimmune diseases like Graves disease and Hashimoto thyroiditis have been reported to be associated with vitiligo. ${ }^{16-18}$ Vitiligo is strongly associated with thyroid disorder. ${ }^{16,17} \mathrm{CD} 8+\mathrm{T}$ cells are specific for melanocyte destruction. ${ }^{19}$ Studies conducted in murine vitiligo model suggests the correlation between the presence of TRP2-180-specific $\mathrm{CD}^{+}$effector T cells and the development of vitiligo. ${ }^{19}$ Studies show that CD8+ $\mathrm{T}$ cell count increased in vitiligo patients compared to normal people. ${ }^{17}$ White patches of vitiligo are often found to be returning after discontinuing treatment. The reason for such recurring of vitiligo spots has been recently found out to be associated with the presence of 'Tissue-resident memory T cells (Trm)'. Trm is formed in the skin in vitiligo and they persist and maintain the disease. ${ }^{20}$ IFN- $\gamma$ has also been found to be intimately associated with the autoimmune disorder mechanism responsible for the occurrence of vitiligo. IFN- $\gamma$ has been found to upregulate the expression of CXCL10. CXCL10 is a chemokine which is known to control the invasion of follicular and epidermal tissues by $\mathrm{CD} 8{ }^{+}{ }$ cells. ${ }^{21}$ 'IFN- $\gamma$, IL-21, and IL-10 co-expression has been found to cause autoimmune vitiligo lesions in Smyth line chickens ${ }^{22}$. Functional association between Trm and recirculating memory $\mathrm{T}$ cells $(\mathrm{Tcm})$ has been studied in murine model. ${ }^{20}$ It has been found that autoantigen in the skin is sensed by both Trm and Tcm even long after stabilization of vitiligo. This results in the production of IFN $\gamma$, CXCL9, and CXCL10 which leads to recurring of the white patches of vitiligo. ${ }^{20}$ Thymic stromal lymphopoietin (TSLP) has been reported to be linked to the pathogenesis of vitiligo. Significantly increased expression of mRNA TSLP has been found in patients of vitiligo compared to that of normal. ${ }^{23}$ IL-15 signalling has also been found to be related to the pathogenesis of vitiligo. ${ }^{24}$ Patients receiving immunotherapy for metastatic melanoma has been reported to develop vitiligo-like depigmentation ${ }^{25}$, thus, establishing the fact that our immune system is intimately associated with the pathogenesis of vitiligo.

IL 17 has been recognized to be intimately associated with the pathogenesis of vitiligo. ${ }^{6-9}$ Studies reveal that drugs and treatments like phototherapy etc. which treat vitiligo, modulate the level of IL-17. ${ }^{8}$ Systemic, tissue, and cellular levels of IL-17 have been reported to be raised in patients with vitiligo. ${ }^{8}$ T helper cell 17 (Th 17) is the one who expresses the cytokine IL 17 has also been shown to be increased in 
patients with vitiligo. ${ }^{6,8}$ The increased levels of IL-17 have also been reported in serum and lesional skin of patients with vitiligo thus establishing the fact that IL 17 plays an important role in the pathogenesis of vitiligo. ${ }^{9}$ Other cytokines like IL21, TGF- $\beta$, (TNF)- $\alpha$ etc. have also been found to be increased in patients with vitiligo.

In vitiligo, melanocytes are targeted by the immune system. Those melanocytes produce 'melanin'-the pigment molecule which exhibits radical scavenging property. The molecules like gp100, MART-1, TRP-2 and TRP-1 etc. and enzymes like tyrosinase etc. are involved in the process of synthesis of melanin. Studies reveal that those molecules are the prime targets of the immune system in vitiligo. In other pathogenic conditions like melanoma also, those molecules are the targets of the immune system. ${ }^{26}$ Studies conducted on Ezyptian population show that there was a significant increase in HLA-A1, A19, B16, CW4, CW 6, DR 4, DR7 and DQ3 in Vitiligo patients compared to that in controls. The study also reveals that increased frequency of HLA-A28, B16, B27, B48, CW2 and DQ3 is remarkable in familial vitiligo. On the other hand, an increased frequency of HLA-A1, CW6 and DR13 is observed in non-familial vitiligo patients. Thus, heterogeneity in the pathogenic process between familial and nonfamilial vitiligo is comprehended in such studies. $^{27}$

Class I and class II major histocompatibility complexes (MHC I \& II) were associated with vitiligo. ${ }^{28}$ Melanocytes have been found to be capable of presenting antigens in the context of MHC class II. ${ }^{28}$ In a study conducted on European-derived Caucasian populations, it has been found that autoimmune vitiligo is strongly associated with MHC class II region SNPs in the vicinity of the $H L A-D R B 1$ and $H L A$ $D Q A 1$ genes. ${ }^{29}$ Thus, there is a strong genetic risk for vitiligo associated with the MHC class II region ${ }^{30}$ which localizes to a haplotype of only three SNPs, which do not encode coding structural variants in the antigen-binding sites of HLA molecules. ${ }^{30}$ Rather, the high-risk MHC class II SNP haplotype is known to be completely intergenic and is located between $H L A-D R B 1$ and $H L A-D Q A 1$, which are present in opposite transcriptional orientations. ${ }^{30}$ Further studies reveal that susceptibility to autoimmune vitiligo in the MHC class II region is associated with the quantitative effect of increased levels of surface expression of HLA molecules ${ }^{30}$ (Fig. 2).

\section{Genes, autoimmunity and vitiligo}

Current studies show that almost all genes associated with autoimmune susceptibility may contribute to the occurrence of vitiligo. Genetics may not be addressed as a separate cause for vitiligo. Rather, genes that cause autoimmunity to melanocytes are the ones responsible for causing vitiligo., ${ }^{9,31}$ Presently strong evidence in support of only a few genes which are responsible for vitiligo occurrence has been presented. Few such genes identified responsible for viti- ligo are HLA, PTPN22, NALP1 and perhaps CTLA4, etc. Those genes are also responsible for autoimmune susceptibility. ${ }^{9,32,33}$ A genetic mutation has also been found to be associated with the occurrence of vitiligo ${ }^{9}$ (Fig.2).

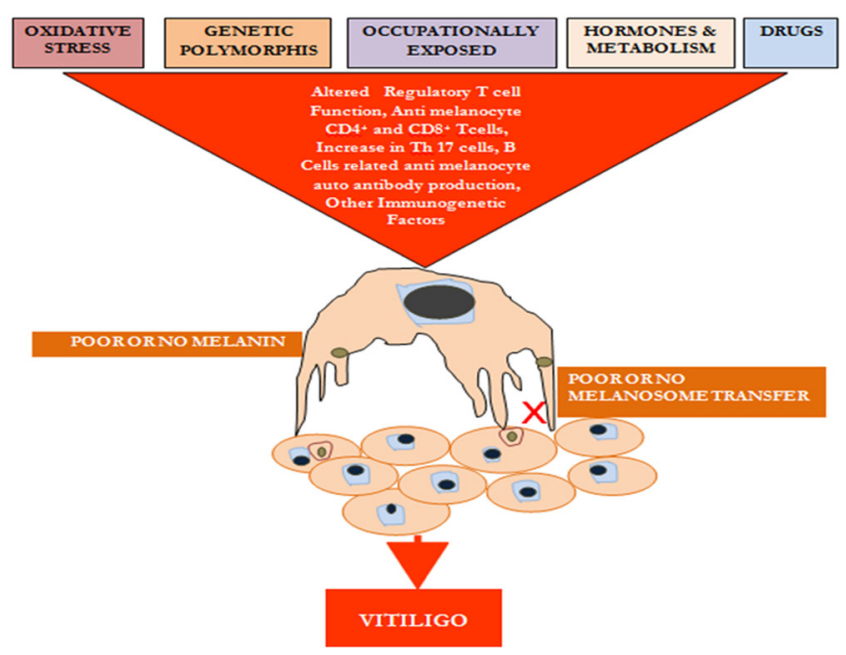

Figure 2: The possible causes and mechanism for the makeover of vitiligo.

\section{Other causes of vitiligo}

Long term consumption of fast food, convenient food and processed food which contain lots of artificial colours, preservatives and other chemical ingredients in them induces oxidative stress in our body which may be considered responsible for the occurrence of vitiligo. Studies show that toxic compound accumulation in cells and altered cellular environment may lead to vitiligo ${ }^{9}$. Also, modernization and our changing lifestyle may be considered responsible for stimulating the occurrence of vitiligo. Studies show that mental stress, depression and anxiety are positively correlated to vitiligo. ${ }^{9}, 34,35$ Mental stress and depression up-regulates formation and release of certain neurohormones which in turn are linked with our immune system. Such immunomodulatory effect of those neurohormones is considered to play a significant role in the initiation of vitiligo. ${ }^{35}$

Certain other chemical compounds have been identified which may induce vitiligo and human are known to get in contact with such chemicals due to occupational purposes. Some such chemicals are p-tert-Butylchatechol, ptert-Butylphenol, p-tert-Amylphenol, Monomethyl ether of hydroquinone (p-methoxy phenol), p-hydroxyanisole), Monoethyl ether of hydroquinone ( $p$-methoxyphenyl), $p$-Phenylphenol, $p$-Octylphenol, $p$-Nonylphenol, $p$-Isopropylcatechol, $p$-Methylcatechol, Butylated hydroxytoluene, Butylated hydroxyanisole, Pyrocatechol (1,2-benxenediol), $B$-Mercaptoethylamine hydrochloride (cysteamine) $\mathrm{N}$-(2-mercaptoethyl)-dimethylamine hydrochloride, Sulfanolic acid, Cystamine dihydrochloride, 3-Mer- 
captopropylamine hydrochloride, $p$-Cresol, Mercurials, Arsenic, Cinnamic aldehyde, $p$-Pheylenediamine, Benzyl alcohol, Azaleic acid, Corticosteroids. Optic preparations like Eserine (physostigmine),Diisopropyl, fluorophoshate, Tiotepa (N, N', N"-triethylene-thiophosphoramide) (Fig.2).

\section{Treatments of vitiligo}

Current trends of treatment of vitiligo involve repigmentation of affected areas. Studies reveal that topical corticosteroids are the primary therapy of vitiligo.$^{36}$ Prostaglandin is used in a topical ointment for repigmentation in treatment of vitiligo ${ }^{37}$ Some drugs have been reported to be effective as well ${ }^{37,38}$. Drugs like Minocycline are in use against vitiligo. ${ }^{38}$ Those drugs work against vitiligo under their antioxidant and immunomodulatory properties. ${ }^{38}$ Dexamethasone has also been studied against vitiligo. ${ }^{39}$ On the other hand, corticosteroids have also been reported to be effective in treating vitiligo ${ }^{40}$ but they bring side effects in patients. ${ }^{41}$ Certain immunomodulatory drugs are in use against vitiligo. Drugs like pimecrolimus which is a calcineurin inhibitor and Tacrolimus, an immunosuppressant that comes from the fungus Streptomyces tsukubaensis, has been reported to heal vitiligo successfully and significantly ${ }^{41}$. Such drugs may have deadly side effects and cause skin cancer etc. ${ }^{41}$ Therefore much use of such drugs should be restricted and should always be continued with strict and regular clinical advice. Simvastatin, a cholesterol-lowering drug, has also been found to effective against vitiligo under its radical scavenging activity and immunomodulatory property ${ }^{42}$. Simvastatin lowers IFN- $\gamma$ and has effects on CD8+ T cells. ${ }^{42}$. Blocking recruitment of Tcm to the skin with FTY720 has been reported to reverse vitiligo. Also, depletion of Tcm using low dose Thy1.1 antibody has been found to reverse disease. These indicate that Trm and Tcm cooperate to maintain vitiligo. ${ }^{20}$ Richmond et al. have also recently reported that vitiligo may be durably reversed by blockade of IL-15 signalling using antibody. ${ }^{24}$

Studies reveal that combination therapy may be effective against vitiligo. ${ }^{43,44}$ Psoralen and light therapy are in use. Psoralen may be consumed orally or applied on the effected area and then the patient is exposed to ultraviolet A or B light bands and has been found to cause the appearance of normal skin colour on the affected areas. ${ }^{43}$ Novel combination therapy against vitiligo using lipid-based nanoemulsion gel using trimethylpsoralen and flavonoid have also been studied and found to be effective. ${ }^{44}$ It has been reported that platelet-rich plasma has a potential role in combined therapy against vitiligo. ${ }^{45}$ Such combination therapies are reported to have better effects in treating vitiligo. ${ }^{43}$ Laser therapy involves the use of UVB A or B bands along with ointments as already discussed. ${ }^{43}$ But these treatments are not always effective and do come with side effects, pains and scars. Hence laser treatment of vitiligo is not much popular.
Another therapeutic option available against vitiligo is surgery. Needling, autologous punch and suction blister grafts, melanocyte keratinocyte transplantation (MKTP) i.e., the non-cultured epidermal cell suspension etc., are the various surgical procedures in use against vitiligo. ${ }^{46,47}$

Depigmentation therapy is also used for vitiligo. It is used in patients with extensive vitiligo. Compounds like Monobenzyl ether of hydroquinone (MBEH) is primarily used in depigmentation therapy. Other phenolic derivatives are also used in depigmentation therapy. Such therapy has local side effects and risk of repigmentation. ${ }^{48-50}$. Certain herbs have been studied against vitiligo and are claimed to be effective in treating vitiligo. ${ }^{49}$ Though vitiligo is not a life-threatening disease as such yet it may have severe social impact and may lead to serious consequences and lead to low self-esteem and paralysed social life. ${ }^{51,52}$ Avoiding the use of cosmetics and chemicals containing phenols ${ }^{15,53}$ and prevention of excessive exposure to sunlight, enough consumption of green leafy vegetables and fruits rich in antioxidants may help to protect us and provide us necessary antioxidants to combat onset and progression of vitiligo. Also, immediate reporting to doctors in the early stage may help stop spreading and also to treat the disease. Cosmetics containing compounds which are phenolic derivatives and are known to cause depigmentation should be strictly avoided to prevent vitiligo. ${ }^{15,50}$ Guanonitrofuracin has also been identified and enlisted as potent chemicals responsible for inducing vitiligo. ${ }^{52,53}$ Systemic medications like Chloroquine and Fluphenazine (Prolixin) are also in the list of chemicals associated with contact/ occupational vitiligo. ${ }^{55}$ In simple words, vitamins, antioxidants, anti-inflammatory and immunomodulatory drugs may be beneficial against vitiligo. Vitiligo is considered as a good model for understanding autoimmune disorders and for trying regenerative medicines. ${ }^{56}$

\section{CONCLUSION}

Our modern food habit seems to have some role for harbouring and nurturing vitiligo which is an oxidative stressmediated autoimmune disorder. There are effective experimental results but yet vitiligo is not curable till date. The disease may be reversed to some extent and maybe stopped from spreading but can't yet being completely cured. We look ahead to a better tomorrow. With more clear knowledge about the autoimmune, oxidative stress-mediated depigmentation disorder, it will be easier to find strong and effective preventive and curative measures against vitiligo. Also, self-education and mass awareness about the etiology, treatments and non-contagious nature of vitiligo is needed to make life comfortable and easy for people affected with vitiligo. 


\section{ACKNOWLEDGEMENT}

Dr. DG and Dr. SG are in WBES and acknowledge the Higher Education Department, Government of West Bengal.

\section{Conflict of Interest}

Authors declare no conflict of interest.

\section{Source of Funding: Nil}

\section{REFERENCES}

1. El-Gayyar MA, Helmy ME, Amer ER, Elsaied MA, Gaballah MA. Antimelanocyte Antibodies: A Possible Role in Patients with Vitiligo. Indian J Dermatol 2020;65(1):33-37.

2. Sehgal VN, Srivastava G: Vitiligo: Compendium of clinicoepidemiological features. Ind J Dermatol Venereol Leprol 2007;73:149-156.

3. What is Vitiligo? https://www.healthline.com/health/skin-disorders/vitiligo-pictures\#: :text=Almost $\% 201 \% 20$ percent $\% 20$ of $\% 20$ the,produce $\% 20$ skin $\% 20$ pigment $\% 2 \mathrm{C} \% 20$ called $\% 20$ melanin. [Accessed on 24.06.2020].

4. Manga P, Elbuluk N, Orlow SJ. Recent advances in understanding vitiligo. F1000Research. 2016;5:F1000 Faculty Rev-2234.

5. Jain A, Mal J, Mehndiratta V, Chander R, Patra SK: Study of Oxidative Stress in Vitiligo. Ind J Clin Biochem 2011;26(1):78-81.

6. Ambrosi A, Espinosa A, Wahren-Herlenius M. IL-17: a new actor in IFN-driven systemic autoimmune diseases. Eur J Immunol 2012; 42(9):2274-2284.

7. Singh RK, Lee KM, Vujkovic-Cvijin I, et al. The role of IL-17 in vitiligo: A review. Autoimmun Rev 2016;15(4):397-404.

8. Bassiouny DA, Shaker O. Role of interleukin-17 in the pathogenesis of vitiligo. Clin Exp Dermatol 2011;36(3):292-297.

9. Yaghoobi R, Omidian M, BagheraniN: Vitiligo: a review of published work. J Dermatol 2011; 38:419-431

10. Picardo M, Bastonini E. A New View of Vitiligo: Looking at Normal-Appearing Skin. J Invest Dermatol 2015; 135(7):17131714.

11. Song P, Li K, Liu L, Wang X, Jian Z, Zhang W, Wang G, Li C, Gao T. Genetic polymorphism of the Nrf2 promoter region is associated with vitiligo risk in Han Chinese populations. J Cell Mol Med. 2016;20(10):1840-1850.

12. Dammak I, Boudaya S, Ben Abdallah F, Turki H, Attia H, Hentati B: Antioxidant enzymes and lipid peroxidation at the tissue level in patients with stable and active vitiligo. Int J Dermatol. 2009; 48(5):476-480.

13. Denman CJ, McCracken J, Hariharan V, et al. HSP70i accelerates depigmentation in a mouse model of autoimmune vitiligo. $J$ Invest Dermatol. 2008;128(8):2041-8.

14. Boissy RE, Liu YY, Medrano EE, et al. : Structural aberration of the rough endoplasmic reticulum and melanosome compartmentalization in long-term cultures of melanocytes from vitiligo patients. J Invest Dermatol 1991;97(3):395-404.

15. Toosi S, Orlow SJ, Manga P: Vitiligo-inducing phenols activate the unfolded protein response in melanocytes resulting in upregulation of IL6 and IL8. J Invest Dermatol. 2012;132(11):26012609.

16. Ai J, Leonhardt MJ, Heymann RW: Autoimmune thyroid disease. Etiology, pathogenesis, and dermatologic manifestations. J Am Acad Dermatol 2003; 48: 641-659.

17. Braverman L, Uitger RD, (eds). Werner and Ingbar are the Thyroids: A Fundamental and Clinic Text, 9th edn. Lippincott Williams and Wilkins, New York 2005: 363.
18. Saleem K, Azim W. Association of Vitiligo with Other Autoimmune Disorders. Diabetes Case Rep. 2016;1:114.

19. You S, Cho YH, Byun JS, Shin EC. Melanocyte-specific CD $8^{+}$ $T$ cells are associated with epidermal depigmentation in a novel mouse model of vitiligo. Clinical and Experimental Immunology 2013;174(1):38-44.

20. Richmond JM, Strassner JP, Rashighi M, Agarwal P, Garg M, Essien KI, Pell LS, Harris JE: Resident memory and recirculating memory $\mathrm{T}$ cells cooperate to maintain disease in a mouse model of vitiligo. J Invest Dermatol 2019 Apr;139(4):769-778.

21. Harris JE, Harris TH, Weninger W, Wherry EJ, Hunter CA, Turka LA. A mouse model of vitiligo with focused epidermal depigmentation requires IFN- $\gamma$ for autoreactive CD8- T-cell accumulation in the skin. J Invest Dermatol 2012; 132(7):1869-76.

22. Shi F, Erf GF. IFN- $\gamma$, IL-21, and IL-10 co-expression in evolving autoimmune vitiligo lesions of Smyth line chickens. J Invest Dermatol 2012;132(3 Pt 1):642-9.

23. Elmasry MF, Nagui NAR, Rashed LA, El Darbi R. Cutaneous expression of thymic stromal lymphopoietin (TSLP) in vitiligo patients: a case-control study. Int J Dermatol 2019 May;58(5):589-592.

24. Richmond J, Strassner JP, Zapata L, Garg M, Riding RL, Reffat MA, et al. Antibody blockade of IL-15 signaling has the potential to durably reverse vitiligo. Sci Transl Med 2018;10(450):eaam7710.

25. Zottarelli F, Giorgione R, Gironi LC, Farinelli P, Sponghini AP, Rondonotti D, Savoia P. Vitiligo-like depigmentation patterns in patients receving immunotherapy for metastatic melanoma: a case series. G Ital Dermatol Venereol. 2018. doi: 10.23736/ S0392-0488.18.06183-7. Online ahead of print.

26. Le Poole I, Luiten R: Autoimmune Etiology of Generalized Vitiligo. Curr Dir Autoimmun 2008;10:227-243 .

27. Elgendy A, Alshawadfy E, Ali E, Wadea N, Khalil K, Alkady $\mathrm{O}$, Alsaidi A. Association of HLA Class I and II Antigens with Vitiligo in Egyptian Population. Mol. Enzymol Drug Targets 2016;2(1):1-6.

28. Cavalli G, Hayashi M, Jin Y, et al. MHC class II super-enhancer increases surface expression of HLA-DR and HLA-DQ and affects cytokine production in autoimmune vitiligo. Proc Natl Acad Sci USA 2016;113(5):1363-1368.

29. Jin Y, Birlea SA, Fain PR, Gowan K, Riccardi SL, Holland PJ, et al. Variant of TYR and autoimmunity susceptibility loci in generalized vitiligo. N Engl J Med 2010; 362(18):1686-1697.

30. Cavalli G, Hayashi G, Jin Y et al. MHC class II super-enhancer increases surface expression of HLA-DR and HLA-DQ and affects cytokine production in autoimmune vitiligo. PNAS 2016;113(5):1363-1368.

31. Spritz RA: The genetics of generalized vitiligo. Curr Dir Autoimmun 2008;10:244-257.

32. Spritz R. The Genetics of Vitiligo. J Invest Dermatol. 2011;131(E1): E18-E20.

33. Le Poole IC, Das PK, Van den Wijngaard RM, Bos JD, Westerhof W: Review of the etiopathomechanism of vitiligo: a convergence theory. Exp Dermatol 1993; 2: 145-153

34. James WD, Berger TG, Elston DM: Andrews. Diseases of the Skin: Clinical Dermatology, 10th edn. Saunders Elsivier, Philadelphia, USA 2006; 860-863.

35. Al-Abadie MSK, Kent G, Gawkrodger DJ: The relationship between stress and the onset and exacerbation of psoriasis and other skin conditions. Br J Dermatol 1994;130:199-203.

36. Bagherani N. Role of Corticosteroids in Treatment of Vitiligo, State of the Art of Therapeutic Endocrinology 2012, Sameh Magdeldin (Ed.), ISBN: 978-953-51-0772-9, InTech, DOI: $10.5772 / 48384$. 
37. Parsad D, Pandhi R, Dogra S, et al. Topical prostaglandin analogue (PGE2) in vitiligo--a preliminary study. Int J Dermatol 2002; 41(12): 942-945.

38. Parsad D, Kanwar A. Oral minocycline in the treatment of vitiligo--a preliminary study. Dermatol Ther 2010;23(3): 305-307.

39. Kanwar AJ, Mahajan R, Parsad D. Low-dose oral mini-pulse dexamethasone therapy in progressively unstable vitiligo. J Cutan Med Surg. 2013; 17(4): 259-268.

40. Lee DY, Kim CR, Lee JH. Recent onset vitiligo treated with systemic corticosteroid and topical tacrolimus: Need for early treatment in vitiligo. J Dermatol 2010; 37(12):1057-1059.

41. Vitiligo. https://www.mayoclinic.org/diseases-conditions/ vitiligo/diagnosis-treatment/drc-20355916 [Accessed on 24.06.2020].

42. Agarwal P, Rashighi M, Essien KI, et al. Simvastatin prevents and reverses depigmentation in a mouse model of vitiligo. J Investig Dermatol 2015;135(4):1080-1088.

43. Shenoi SD, Prabhu S: Photochemotherapy (PUVA) in psoriasis and vitiligo. Ind J Dermatol Venereol Leprol 2014;80:497-504.

44. Shivasaraun UV, Sureshkumar R, Karthika C, Puttappa N. Flavonoids as an adjuvant in psoralen based photochemotherapy in the management of vitiligo/leucoderma. Med Hypotheses 2018; 121:26-30.

45. Shih S. Platelet-rich plasma: Potential role in combined therapy for vitiligo. Dermatol Ther. 2019: 32(1):e12773.
46. Mulekar SV, Isedeh P. Surgical interventions for vitiligo: an evidence-based review. Br J Dermatol. 2013;169(3):57-66.

47. Sahni K, Parsad D, Kanwar AJ, et al. Autologous non-cultured melanocyte transplantation for stable vitiligo: can be suspending autologous melanocytes in the patients' serum improve repigmentation and patient satisfaction? Dermatol Surg. 2011; 37(2):176-182.

48. Gupta D, Kumari R, Thappa DM: Depigmentation therapies in vitiligo. Ind J Dermatol Venereol Leprol 2012;78:49-58.

49. Mahjour M, Khoushabi A. A Review Article About Some Herbs Affecting Vitiligo Based on Avicenna's Canon. Curr Drug Discov Technol. 2020;17(2):133-137.

50. Papadopoulos L, Bor R, Legg C. Coping with the disfiguring effects of vitiligo: a preliminary investigation into the effects of cognitive-behavioural therapy. Br J Med Psychol 1999;72:385396.

51. Grimes PE, Miller MM. Vitiligo: Patient stories, self-esteem, and the psychological burden of disease. Int J Women's Dermatol 2018;4(1):32-37.

52. Boissy R, Manga P. On the etiology of contact/occupational vitiligo. Pigm Cell Res 2004;17:208-214.

53. Picardo M, Dell'Anna M, Ezzedine K. Vitiligo. Nat Rev Dis Primers 2015;1:15011. 